\title{
INCLUYENDO EL AGOTAMIENTO DE LOS RECURSOS NATURALES EN LAS CUENTAS NACIONALES: EVIDENCIA PERUANA DEL PERIODO 1994-2011
}

\author{
INCLUDING THE DEPLETION OF NATURAL RESOURCES IN NATIONAL ACCOUNTS: \\ PERUVIAN EVIDENCE OF YEAR 1994-2011
}

\author{
${ }^{1}$ Carlos Enrique Orihuela Romero
}

\begin{abstract}
Resumen
El objetivo del estudio es determinar una medida de ingreso nacional peruano que considere la pérdida del capital natural. Adicionalmente se incluyó el capital humano y degradación ambiental. Para ello se utilizó el modelo de Hamilton (1994, 2000). Los resultados para el periodo 1994-2011- indican que las medidas de ingreso tradicionales han sido sobrestimadas en un rango de 4-10\% dependiendo del año. Se concluye que la economía peruana se encuentra en la senda óptima del crecimiento (a pesar del agotamiento de los recursos naturales), lo cual es fruto -básicamente- del buen manejo económico, de la favorable coyuntura mundial de precios de los metales y de la riqueza geológica peruana. Así, para potenciar las posibilidades de crecimiento futuro se requiere revisar e implementar una política integral sobre los recursos naturales, y en general de los recursos de la economía.
\end{abstract}

Palabras clave: cuentas verdes, PIB, depreciación.

\begin{abstract}
The aim of the study is to determine a measure of national income, which considers not only the loss of natural capital but human capital and environmental degradation. The results for the 1994-2011 indicate that traditional measures of income have been overestimated (4-10\%) depending on the year. It is concluded that the Peruvian economy is on the optimal path (despite the depletion of natural resources). Thus, to enhance opportunities for future grow this required to review and implement a comprehensive policy on natural resources.
\end{abstract}

Key words: green accounting, GDP, depreciation.

\section{Introducción}

Desde tiempos de la colonia, la economía peruana ha dependido fundamentalmente de la extracción y venta de recursos naturales. Esta dependencia ha sido creciente a lo largo de los siglos, la cual en los últimos tiempos se ha acrecentado con la subida de los precios metales. Ello se ha traducido en mayores ingresos al Estado y una creciente dinámica alrededor de los sectores extractivos. Al mismo tiempo significa que los recursos naturales, principalmente hidrocarburos y metales, serán extraídos y eventualmente agotados, y por ende es necesario no solo su uso óptimo sino también reinvertir eficientemente sus rentas.

Una gestión inadecuada de los recursos naturales podría peligrar la satisfacción de necesidades de las próximas generaciones: ¿Cuáles son las implicancias del eventual agotamiento de estos recursos para el crecimiento económico en el largo plazo? Por ello, es necesario evaluar el desempeño económico de estos sectores. Una forma de medir este desempeño y de los sectores que la conforman es mediante las medidas de ingreso tradicionales, tales como el producto interno bruto y el producto nacional neto (de ahora en adelante, PIB y PNN respectivamente). En el caso de los sectores extractivos, los PIB y PNN sectoriales consideran la extracción de recursos naturales solo como ingreso y no como consumo de capital. Si tales recursos constituyen activos naturales entonces también deben ser sujetos de depreciación o agotamiento, detalle que es omitido por el actual Sistema de Cuentas Nacionales. Por ende, las medidas de ingreso tradicionales no reflejan el real desempeño de los sectores extractivos.

Esto es particularmente importante en un país como el Perú, cuyas exportaciones de recursos naturales concentraron más del $77 \%$ del total (BCRP, 2013). Si el Estado considera una medida de ingreso sobrestimada, su decisión podría conllevar a políticas inadecuadas, como por ejemplo, priorizar la extracción de un recurso natural en desmedro, quizá, de su conservación.

El objetivo de esta investigación es incorporar la depreciación natural y degradación ambiental en las cuentas nacionales a fin de obtener una medida de ingreso nacional peruana más apropiada para la toma de decisiones. El periodo de estudio es 1994-2011. 


\section{Marco conceptual}

\section{Ingreso Nacional Neto y consumo de capital natural}

Weitzman (1976) demostró que, bajo una senda optima de una economía dinámica y competitiva, el PNN medido como la suma del consumo e inversión de un periodo dado, iguala al valor presente del consumo a lo largo de la senda en mención. Este producto debe incluir los cambios en todos los stocks de capital de la economía, incluyendo los recursos naturales. En otras palabras, el verdadero ${ }^{2}$ producto nacional es simplemente la cantidad que un planificador elegiría en cada periodo a fin de maximizar el valor presente del consumo.

Este valor puede ser entendido no solo como una medida de ingreso sino también de riqueza (Hamilton, 1996). El hallazgo de Weitzman ha sido entonces el enlace entre el producto nacional neto y la medición apropiada del consumo de otros capitales. Una implicancia es que el PNN debería ser no decreciente, lo cual garantizaría un consumo sostenible o una mejora del bienestar social. Adicionalmente, el cálculo adecuado del producto nacional neto permitiría evaluar la fracción del ingreso convencional atribuido al agotamiento de recursos naturales. Esto a su vez, permite establecer una tasa de crecimiento de la economía más precisa.

Cabe mencionar que aun cuando una medida de ingreso sea inclusiva y adecuadamente estimada, no sería un indicador de desarrollo sostenible puesto que tal medida solo ofrece una corrección o ajuste del ingreso en un momento dado y no permite inferir mucho sobre el desarrollo futuro. ${ }^{3}$ De esta forma, el PNN sería una buena medida de bienestar social, aun cuando pueda no ser un buen indicador de sostenibilidad (Aronsson et al., 1997).

\section{Cuentas Nacionales y Cuentas Verdes (Ambientales)}

El Sistema de Cuentas Nacionales (SCN) provee medidas ampliamente utilizadas como el PIB y el PNN, siendo la primera la más utilizada, no obstante, la segunda brinda una mejor imagen del ingreso, al deducir el consumo de capital fijo. Al margen de las omisiones clásicas de estas medidas $^{4}$, el SCN omite indicadores como el agotamiento de los recursos naturales (depreciación natural) y la degradación o daño ambiental.

Una forma de incorporar tal agotamiento y degradación es mediante el Sistema de Contabilidad Ambiental y Económico Integrado (SEEA, por sus siglas en ingles). No obstante, este nuevo sistema aun no ha podido ser generalizado debido, entre otras causas, a las limitaciones de información y capacidades, poco interés

${ }^{2} \mathrm{El}$ término verdadero, en este contexto, es equivalente a "inclusivo", en el sentido que debería considerarse todas los tipos de capital que conforman la economía

${ }^{3}$ Por ello, Dasgupta y Mäler (2001) propusieron el indicador inversión genuina como el más apropiado para evaluar el desarrollo sostenible.

${ }^{4} \mathrm{E}$ PIB solo registra las retribuciones a factores de la producción que son transados en el mercado. de las autoridades, y sobre todo, falta de consenso sobre las metodologías de valoración de la degradación y el agotamiento de los recursos naturales. Por ello, el SEEA es prácticamente utilizado como un sistema paralelo aunque eventualmente complementario para la toma de decisiones.

La inclusión de la depreciación natural y degradación ambiental en las medidas tradicionales de ingreso constituyen medidas de ingreso verde. La literatura de estas últimas medidas es relativamente abundante (aunque Latinoamérica es la excepción). Los autores coinciden en la corrección del PNN (Weitzman, 1976; Repetto et al. 1989; Hartwick, 1990) en el sentido que éste debe incluir otras formas de capital (natural y humano) a fin de obtener una medida apropiada del ingreso.

\section{Marco metodológico}

Hamilton (2000) propone un modelo de ingreso (ingreso verde) que incluye no solo la depreciación natural sino también la degradación ambiental generada por la extracción de un recurso natural, el cual es comentado a continuación.

Se asume una simple economía cerrada ${ }^{5}$ con un solo recurso utilizado como insumo en la producción de un bien agregado que puede ser consumo, invertido en otros activos, como capital humano o para mitigar la contaminación de manera que $F(K, R, N)=C+K+a+m$, donde ${ }^{F}$ es el nivel de producción, el cual depende del capital artificial ${ }^{K}$, del recurso utilizado $\left({ }^{\mathrm{R}}\right)$ y del capital humano $\left({ }^{N}\right)$, siendo ${ }^{a}$ el gasto de mitigar la contaminación, $\mathrm{y}^{m}$ la inversión en capital humano (gastos corrientes en educación).

La función $q(m)$ transforma los gastos de educación en capital humano que no se deprecia, de manera que ' $N=q(m)$, lo cual equivale a un incremento en el capital humano. El insumo trabajo es fijo y por ende no es incluido en la función de producción.

Las emisiones de contaminantes $\left({ }^{e}\right)$ dependen no solo de los niveles de producción y mitigación (de manera que $e=e(F, A)$ ) sino también del nivel de contaminantes acumulados en un stock ${ }^{X}$ de tal forma que ' $X=e-d(X)$ donde ${ }^{\mathrm{d}}$ es la cantidad de disipación natural del stock contaminante. El flujo de servicios ambientales $B$ está negativamente relacionado al tamaño del stock de contaminación, de manera que $B=a(X)$ siendo $a_{X}<0$.

El recurso $S$ crece a una tasa $g$ y es agotado por una extracción ${ }^{R}$, de forma que ' $S=-R+g(S)$. Se asume que la utilidad de los consumidores es una función del consumo y servicios ambientales, de tal forma que $U=U(C . B)$.

Asumiendo que el planificador social desea maximizar la riqueza, se desprende que la función objetivo está dada por (1):

${ }^{5}$ Hamilton (2000) incorpora una identidad adicional al modelo para considerar el sector externo, sin embargo, ello no cambia el resultado del modelo para economía cerrada. 


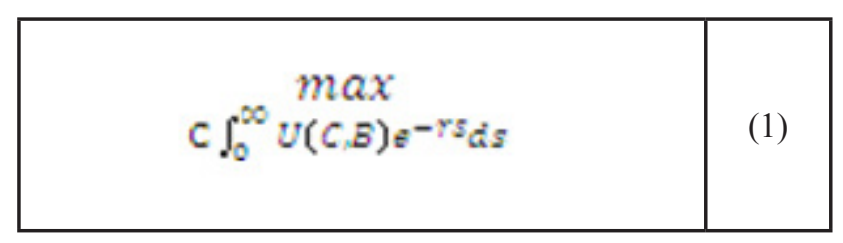

sujetoa cuatro restricciones:

\begin{tabular}{|c|c|}
\hline 'K=F-C-a & (2) \\
\hline$\dot{X}=e-d$ & (3) \\
\hline 'S $=-R+\mathrm{g}$ & $(4)$ \\
\hline$' N=q(m)$ & $(5)$ \\
\hline
\end{tabular}

Para este modelo, el valor corriente de la función Hamiltoneana, la cual es maximizada en cada punto del tiempo, está dada por:

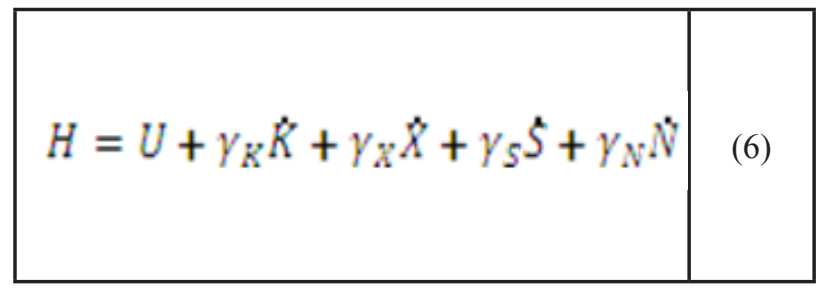

donde $\gamma_{K}, \gamma_{X}, \gamma_{S}, y \gamma_{N}$ son los precios sombra (en útiles) del capital, contaminación, recursos y capital humano, respectivamente. Resolviendo el problema se obtiene una expresión equivalente al PNN corregido (7):

$$
\begin{aligned}
& P N N A=C+\dot{K}-\left(1-b e_{F}\right) F_{R}(R-g)- \\
& b(e-d)+{ }^{q} / q_{m}
\end{aligned}
$$

Los dos primeros términos del lado derecho $(C+K$ ) equivalen al PNN convencional $(P N N)$. Nótese que $1 /$ capital humano. Asimismo, $b$ es el costo marginal de mitigar la contaminación, el cual equivale al costo marginal social de emisiones contaminantes y también al nivel de impuesto pigouviano para maximizar bienestar (Hamilton, 1996). Definiendo renta marginal del recurso como $n \equiv\left(1-b e_{F}\right) F_{R}$, entonces (7) se reescribe como (8):

$$
P N N A=P N N-n(R-g)-b(e-d)+{ }^{q} / q_{m}
$$

De esta forma, el PNN convencional debe ser ajustado mediante la resta del agotamiento neto de recursos naturales y los daños marginales de la acumulación neta de contaminación pero incluyendo las inversiones en capital humano (quinto término del lado derecho de (8)).
Una limitante de este modelo es la omisión de los nuevos descubrimientos, lo cual es relevante para una economía que depende en gran medida de los recursos no renovables. El aumento del stock de estos recursos también debería reflejarse en la medida de ingreso.

Por ello, y basado en Hamilton (1994), se adiciona una nueva restricción al modelo, $\dot{M}=D$ siendo $D$ los nuevos descubrimientos del recurso no renovable y $\dot{M}$ son los descubrimientos acumulativos. De esta forma, se define $h(D, M)$ como la función de costos de descubrimiento, el cual no solo depende del nivel de descubrimientos corrientes sino también de los racmibrimientos acumulados (donde ${ }^{h} D>0$ y ${ }^{h} M>0$ ) y $f(R)$ es la función de costo de extracción del recurso no renovable.

Incorporando al modelo la restricción adicional y adicionando las dos últimas funciones en (2), el resultado será (9):

\begin{tabular}{|l|l|}
\hline$P N N C=P N N-n(R-g)-$ & (9) \\
$b(e-d)+{ }^{q} / q_{m}+h_{D} D$ &
\end{tabular}

El último termino del lado derecho de (9) es equivalente a los nuevos descubrimientos valorados por su costo marginal de descubrimiento. Evidentemente, para el caso de recursos no renovables se asume que $g \equiv 0$ y $h_{D} D \equiv 0$ Cabe señalar que generalmente no hay información disponible sobre nuevos descubrimientos por metal o hidrocarburo ni su respectivo costo marginal. Una gruesa aproximación del término $\left(h_{D} D\right)$ es la inversión en exploración, atajo utilizado por Figueroa et al. (2002). Esta inversión ya es deducida en el $P N N$, de manera que incorporarla generaría un problema de doble contabilidad, sin embargo debe considerarse que esta inversión es solamente una gruesa aproximación a un valor buscado y por ende, no debe ser considerado como tal.

La expresión (9) es utilizada para el caso de los principales sectores extractivos de recursos naturales del Perú. Esta priorización se efectúa conforme a su contribución al PIB total. Solo se considera el sector minería e hidrocarburos. No se incluye el sector forestal puesto que su contribución al PIB es mínima. Al margen de ello, no se dispone de información sobre precios sombra (o que permita inferirla) ni sobre la evolución de los stocks forestales.

Tampoco se considera la pesca marítima (de anchoveta) puesto que si bien los niveles de extracción están disponibles, no se tiene información sobre la tasa de crecimiento anual de la biomasa. Es de esperar que las capturas (extracción) anuales permitidas por la normatividad sectorial ${ }^{6}$ hayan sido obtenidas en base a

${ }^{6}$ El Decreto Legislativo 1084 establece la Ley sobre límites máximos de captura por embarcación, cuyo objetivo es establecer el mecanismo de ordenamiento pesquero aplicable a la extracción de los recursos de anchoveta y anchoveta blanca, modernización y eficiencia, promover su desarrollo sostenido como fuente de alimentación, empleo e 
criterios biológicos (tasa de regeneración) de manera que el crecimiento neto sea cercano a cero. De ser así, la depreciación/apreciación seria mínima, y su eventual incorporación en los resultados de este estudio no debería ser significativa. ${ }^{7}$

Este estudio no considera la depreciación del terreno agrícola debido a la falta de información sobre tasas de erosión en las zonas agrícolas más relevantes del país (en función a su ingreso). ${ }^{8}$ Dadas estas omisiones, la medida de ingreso a obtener en este estudio constituye una primera aproximación al ingreso verde.

Para la aplicación de la expresión (9) se requiere información del PNN, la cual está disponible en INEI (2013a), no obstante para completar gran parte de la expresión (9) fue necesario efectuar diversas estimaciones. A continuación se comenta la forma de estimación de los componentes (precio sombra, niveles de extracción, valor de los nuevos descubrimientos, entre otros) de la expresión (9) por cada sector en análisis.

\section{Sector minero}

La actividad minera peruana es polimetálica basándose en la extracción, concentración/refinación (según sea el metal) y venta de oro, plata, cobre, plomo, zinc, estaño, y hierro, los cuales constituyen los principales metales comerciales que representan el grueso de los ingresos y valor agregado sectorial.

Si bien la información sobre los niveles de extracción de tales metales está disponible en MINEM (2012), no existe información de los respectivos precios sombra, de manera que éstos tuvieron que ser estimados.

Los precios de mercado de los metales de contenido fino están disponibles, sin embargo, el grueso de la producción minera metálica peruana corresponde a concentrados. Además, algunas empresas mineras peruanas venden diferentes productos de un mismo metal, y por ende, éstos tienen diferentes precios, los cuales tampoco están disponibles, y por tanto, tuvieron que ser estimados.

\section{Precios}

Para superar este problema, se calculó un precio promedio ponderado por cada metal, el cual equivale a la división del valor de la exportación del metal entre la sumatoria del volumen de sus productos exportados. El BCRP (2013) proporciona la información que permite estimar estos precios para los metales en análisis durante todo el periodo en estudio.

Puesto que lo importante es obtener el precio sombra

ingresos, y asegurar un aprovechamiento responsable de los recursos hidrobiológicos, en armonía con la conservación de la biodiversidad. De ser así, las cuotas de captura deben tener por objetivo la sostenibilidad del recurso pesquero, lo cual se logra con la tasa de captura máxima sostenible.

${ }^{7}$ Para el año 2012, el PIB pesquero peruano concentró apenas el 0,37\% del PIB total (BCRP, 2013).

${ }^{8} \mathrm{Sin}$ embargo, su incorporación podría resultar significativa puesto que el sector agropecuario concentró el año 2012 el 7\% del PIB total (BCRP, 2013) (diferencia entre precio de mercado y costo marginal de extracción) y no el valor en sí del precio de mercado y/o costo marginal de extracción, resulta preferible utilizar este precio ponderado. Además, el precio sombra refleja el beneficio marginal percibido por la extracción del metal, lo que no se puede obtener usando solo el precio de mercado.

\section{Costo marginal de extracción}

Ni los costos medios ni los costos marginales de extracción estuvieron disponibles. Para estimarlos fue necesario construir -mediante técnicas econométricas- funciones de costo total por metal para luego derivar el costo marginal respectivo por año. Para inferir el costo total anual de extracción se utilizó la diferencia de ingresos (por ventas) y beneficios (antes de impuestos) de las empresas mineras más representativas en la producción de cada metal. Esta información fue obtenida principalmente de las memorias de empresas mineras y de su información financiera disponible.

Lo que debería incluirse en el precio sombra minero (o deducirse del ingreso verde) es el valor del daño ambiental ocasionado por la extracción minera y no los costos de mitigación asociados (Vincent y Rozali, 2005). Sin embargo, dada la disponibilidad de información, solo fue posible considerar los costos de mitigación como una gruesa aproximación de los daños mencionados. Estos costos ya están incluidos en los costos totales de extracción de las empresas mineras que fueron consideradas en este estudio. La forma de estimación de la función de costo total de extracción por cada metal es presentado en Orihuela (2012).

\section{Sector hidrocarburos}

El valor agregado del sector hidrocarburos está vinculado a la extracción y venta de petróleo crudo (líquidos) y gas natural. Para el caso del petróleo crudo, Ego-Aguirre (2012) estimó la función de costo total de la industria petrolera peruana para el periodo 1996-2009 en la zona costera y selva, en base a la cual es factible inferirse los costos marginales de extracción por zona.

Puesto que el estudio de Ego-Aguirre (2012) ofrece información financiera sectorial agregada, es factible inferir un precio promedio ${ }^{2}$ PETROLEO, a partir de los ingresos sectoriales $\left({ }^{I T} P E T R O L E O\right)$ y la producción de petróleo, QPETROLEO. Formalmente,

$$
P_{\text {PETROLEO }}=\frac{\text { ITPETROLEO }}{\text { QPETROLEO }_{\text {PET }}}
$$

En el caso del gas natural, este ha sido extraído de forma conjunta con el petróleo. A partir de la puesta en marcha del Proyecto Camisea del año 2004, el gas natural es 
obtenido como un producto principal. La mayor parte de la producción nacional de gas proviene del proyecto en mención, de manera que la información de sus precios y costos marginales de extracción es representativa.

Conforme a Apoyo (2007) el valor actual de los beneficios proyectados para el periodo 2004-2010 asciende a 5322 US\$ millones, los cuales comprenden los ahorros generados en los consumidores directos del gas y los consumidores de energía eléctrica. ${ }^{9}$ Naturalmente, deben adicionarse los beneficios vinculados a la extracción de gas que son percibidos por la empresa (Pluspetrol). Ambos beneficios actualizados equivalen al precio sombra multiplicado por la cantidad de gas extraída durante el periodo señalado.

Para convertir los resultados de Apoyo (2007) en cifras anuales, fue necesario calcular la anualidad correspondiente mientras que la información sobre beneficios de la empresa fue obtenida a partir de sus memorias anuales.

\section{Degradación ambiental}

En general, la degradación ambiental puede agruparse sobre dos grandes componentes ambientales: aire y agua. En el Perú poco se ha estudiado sobre el costo económico de la degradación de estos componentes como resultado de la extracción de recursos naturales, principalmente, mineros, hidrocarburos e incluso forestales.

Este costo se mide generalmente a través del impacto en la salud asociado a los niveles de concentración de ciertos elementos contaminantes en el aire como $\mathrm{PM}_{10}, \mathrm{SO}_{x}, \mathrm{NO}_{x}$, etc. Lo común ha sido aplicar el método transferencia de beneficios. Ejemplos son: Sánchez (1998), Ostro (2004), y Croitoru (2010) para Chile, Estados Unidos y Jordania, respectivamente.

En el Perú se han realizado pocos estudios al respecto, limitándose a calcular el costo económico anual para Lima Metropolitana debido a la concentración de cierto nivel de $\mathrm{PM}_{10}{ }^{10}{ }^{10}$ Para el año 2001, Kröger (2002) estimo el costo de reducir la concentración de $\mathrm{PM}_{10}$ de 119 a 50 $\mathrm{ug} / \mathrm{m}^{3}$. Miranda (2006) hizo lo mismo para el año 2005, estimando la reducción de esa concentración de 93 a 50 $\mathrm{ug} / \mathrm{m}^{3}$.

Aparte del $\mathrm{PM}_{10}$, Loyola y Soncco (2007) calculan el beneficio económico de la reducción de plomo en la sangre de la población infantil en una localidad de la provincia Callao. Con respecto al componente agua, solo Herrera y Millones (2011) calculan el costo de la contaminación ambiental minera sobre los recursos hídricos en el Perú para los años 2008 y 2009.

\section{Efectos de la concentración de $P M_{10}$ en la salud}

${ }^{9}$ Nótese que estos beneficios equivalen a las externalidades positivas del sector hidrocarburos, las cuales deben ser consideradas en el precio sombra.

${ }^{10}$ Presumiblemente escasez de literatura nacional sobre costos ambientales radique en la falta de información sobre funciones dosis-respuesta en el campo de la salud o recursos hídricos. Otra causa podría ser la falta de estadísticas o evolución temporal de monitoreos ambientales en las principales ciudades.
Los efectos de la concentración de $\mathrm{PM}_{10}$ la salud humana se estiman en base a las funciones dosis-respuesta que evalúan la relación entre el incremento del número de casos anuales de mortalidad y morbilidad ante un incremento de $\mathrm{PM}_{10}$.

\section{Efectos de la contaminación ambiental sobre el agua}

$\mathrm{Si}$ bien se conocen los impactos que generan las actividades extractivas (básicamente, minería e hidrocarburos), poco se ha trabajado sobre la estimación económica de tales impactos. Una contribución ha sido el estudio de Herrera y Millones (2011), quienes basados en una muestra representativa de empresas mineras y empleando el modelo de la frontera de posibilidades de la producción estimaron que el costo de la contaminación hídrica para el año 2008 fue 815 US\$ millones corrientes (= 359 US\$ millones constantes 1994).

La limitación de este resultado es que no valora el impacto de la contaminación hídrica sobre el ambiente per se(es decir, el daño ambiental) sino el costo de oportunidad hipotético de los agentes mineros por cumplir la normativa sectorial (límites máximos permisibles). Dada la ausencia de mayor información, este resultado fue utilizado, extrapolándosele al resto del periodo mediante regla de tres simple a partir del PIB real minero. Se reconoce que el valor obtenido sobrestima el verdadero valor de la degradación ambiental.

\section{Capital humano}

La información de inversión en capital humano está disponible. Una gruesa aproximación es el gasto que destina el Estado para la educación en el Perú. Sin embargo, esta técnica omite las inversiones realizadas por agentes privados, como aquellas realizadas por los sectores minería e hidrocarburos en conocimiento geológico (gasto en exploraciones) lo cual ya estaría internalizado en la expresión (9). El gasto estatal en educación (en US\$ 1994) durante el periodo de estudio oscilo entre 5-9 millones de dólares, de manera que su contribución a (9) no es significativa. ${ }^{11}$

\section{Resultados}

Todos los resultados fueron convertidos a dólares constantes del año 1994 usando el deflactor implícito del PIB sectorial correspondiente (Tabla 1).

La mayor parte del valor de la depreciación natural corresponde a la depreciación minera. Nótese que el valor de la degradación ambiental es proporcionalmente reducido en comparación a cualquier tipo de depreciación natural. Esta brecha debería reducirse en la medida que se midan y valoren (económicamente) los efectos ambientales generados por las actividades extractivas. Debe recordarse que la metodología aplicada para valorar la degradación ambiental, si bien es consensuada en la

\footnotetext{
${ }^{11}$ Como cualquier tipo de capital, también debería ser sujeto de depreciación, no obstante su incorporación no sería significativa.
} 
literatura, es una gruesa extrapolación de otras realidades. El valor económico de la depreciación minera tiende a reducirse en términos absolutos y relativos en los últimos años del periodo en análisis, lo cual es explicado por la menor extracción y venta del grueso de metales comerciales, así como con el aumento de los ingresos de otras actividades económicas, como el comercio, el cual ha desplazado los ingresos de la minería, de manera que esta aparente menor dependencia de la economía con respecto a la actividad minera es engañosa.

Tabla 1 Valor de la depreciación natural por sector y degradación ambiental (1994 US\$ millones).

\begin{tabular}{lccccccc}
\hline \multirow{2}{*}{ Año } & \multicolumn{3}{c}{ Depreciación natural } & \multicolumn{3}{c}{ Degradación ambiental } \\
\cline { 2 - 8 } & Minera & Petróleo & Gas & Total & Aire & Agua & Total \\
\hline 1994 & 1395 & 790 & $\mathrm{Nd}$ & 2185 & 20 & 136 & 155 \\
1995 & 1679 & 756 & $\mathrm{Nd}$ & 2435 & 19 & 145 & 165 \\
1996 & 2797 & 726 & $\mathrm{Nd}$ & 3523 & 19 & 155 & 175 \\
1997 & 1750 & 606 & $\mathrm{Nd}$ & 2355 & 32 & 172 & 204 \\
1998 & 2888 & 375 & $\mathrm{Nd}$ & 3263 & 34 & 179 & 214 \\
1999 & 2670 & 440 & $\mathrm{Nd}$ & 3110 & 32 & 208 & 240 \\
2000 & 2398 & 542 & $\mathrm{Nd}$ & 2940 & 38 & 267 & 305 \\
2001 & 3054 & 384 & $\mathrm{Nd}$ & 3438 & 37 & 239 & 276 \\
2002 & 3366 & 347 & $\mathrm{Nd}$ & 3713 & 18 & 270 & 288 \\
2003 & 3544 & 336 & $\mathrm{Nd}$ & 3880 & 46 & 287 & 333 \\
2004 & 2803 & 376 & 404 & 3583 & 51 & 302 & 353 \\
2005 & 3765 & 444 & 410 & 4619 & 70 & 324 & 394 \\
2006 & 2723 & 506 & 410 & 3638 & 163 & 327 & 490 \\
2007 & 4015 & 544 & 412 & 4971 & 60 & 335 & 395 \\
2008 & 5711 & 750 & 410 & 6871 & 99 & 359 & 459 \\
2009 & 4625 & 449 & 414 & 5488 & 67 & 357 & 424 \\
2010 & 3803 & 587 & 423 & 4813 & 40 & 346 & 387 \\
2011 & 3978 & 558 & 427 & 4963 & 25 & 338 & 363 \\
\hline
\end{tabular}

Nd: no disponible

No se incluye la contribución del capital humano la cual oscila entre US\$ 4-9 millones.

Los resultados de este estudio permiten inferir que una fracción equivalente al $4-10 \%$ del ingreso convencional (PIB) ha correspondido al agotamiento de los recursos naturales y la degradación ambiental (Tabla 2). Esta fracción ha sido decreciente en los últimos años del horizonte analizado, lo cual es explicado por el mayor aporte de otras actividades económicas en el ingreso y no necesariamente debido a una mejor eficiencia en el uso de los recursos naturales.

Tabla 2. Medidas tradicionales y corregidas (US\$ 1994 millones).

\begin{tabular}{ccccccc}
\hline \multirow{2}{*}{ Año } & \multicolumn{2}{c}{ Medidas tradicionales } & \multicolumn{2}{c}{ Medidas corregidas } & \multicolumn{2}{c}{$\begin{array}{c}\text { Sobrestimación (\%) } \\
\text { del }\end{array}$} \\
\cline { 2 - 6 } & PIB & PNN & PIBC & PNNC & PIB & PNN \\
\hline 1994 & 44808 & 42164 & 42239 & 39595 & 6.08 & 6.49 \\
1995 & 48665 & 45648 & 45844 & 42827 & 6.15 & 6.59 \\
1996 & 49891 & 46648 & 46039 & 42796 & 8.37 & 9.00 \\
1997 & 53315 & 49850 & 50637 & 47172 & 5.29 & 5.68 \\
1998 & 52965 & 49310 & 49546 & 45892 & 6.90 & 7.45 \\
1999 & 53449 & 49761 & 49961 & 46273 & 6.98 & 7.54 \\
2000 & 55026 & 51174 & 51598 & 47746 & 6.64 & 7.18 \\
2001 & 55144 & 51008 & 51255 & 47119 & 7.59 & 8.25 \\
2002 & 57910 & 53625 & 53710 & 49425 & 7.82 & 8.50 \\
2003 & 60248 & 55910 & 55799 & 51462 & 7.97 & 8.64 \\
2004 & 63246 & 58756 & 59109 & 54619 & 7.00 & 7.57 \\
2005 & 67564 & 62767 & 62384 & 57587 & 8.30 & 9.00 \\
2006 & 72793 & 67771 & 68447 & 63424 & 6.35 & 6.85 \\
2007 & 79276 & 73806 & 73720 & 68250 & 7.54 & 8.14 \\
2008 & 87048 & 81216 & 79512 & 73679 & 9.48 & 10.23 \\
2009 & 87798 & 81828 & 81969 & 75999 & 7.11 & 7.67 \\
2010 & 95520 & 89215 & 90547 & 84243 & 5.49 & 5.90 \\
2011 & 102122 & 95586 & 97199 & 90663 & 5.07 & 5.43 \\
\hline
\end{tabular}


Los resultados de este estudio parecerían ser relativamente bajos en comparación a otros estudios (Tabla 3). Esto puede ser explicado por algunos motivos.

Tabla 3. Medidas tradicionales y corregidas en otros países.

\begin{tabular}{|c|c|c|c|c|c|c|c|}
\hline País & Periodo & Sector & $\%$ del PIB & Método & Autor & $\begin{array}{l}\text { Recursos } \\
\text { naturales }\end{array}$ & Ambiente \\
\hline Brasil & $1970-1988$ & Todos & $9-89 * *$ & $\mathrm{PN}, \mathrm{CU}$ & Young y da Motta (1995) & $\mathrm{x}$ & \\
\hline Indonesia & $1971-1984$ & Todos & $25 * *$ & PN & Repetto et al. (1989) & & \\
\hline Portugal & $1990-2005$ & Todos & $15^{* *}$ & $\mathrm{PN}$ & Mota et al. (2010) & $\mathrm{x}$ & $\mathrm{x}$ \\
\hline Kuwait & $1977-1980$ & Todos & $60-70 * *$ & $\mathrm{PN}$ & Stauffer (1986) & $\mathrm{x}$ & \\
\hline Noruega & $1978-1981$ & Todos & $2-8 * *$ & PN & Stauffer (1986) & $\mathrm{x}$ & \\
\hline Chile & $1985-1997$ & Todos & $3 * *$ & $\mathrm{PN}$ & Figueroa y Calfucura (2002) & $\mathrm{x}$ & $\mathrm{x}$ \\
\hline Chile & 1977-1996 & Minería & $20-40 *$ & $\mathrm{PN}$ & Figueroa et al. (2002) & $\mathrm{x}$ & \\
\hline Australia & 1988-1992 & Minería & $29-45^{*}$ & $\mathrm{CU}$ & Common y Sanyal (1998) & $\mathrm{x}$ & \\
\hline Perú & $1979-1993$ & Minería & $1-30 *$ & $\mathrm{PN}, \mathrm{CU}$ & Pasco-Font et al. (1995) & $\mathrm{x}$ & \\
\hline Perú & $1992-2001$ & Minería & $26-38 *$ & PN & Orihuela y Ponce (2004) & $\mathrm{x}$ & \\
\hline Perú & $1992-2006$ & Minería & $51-64 *$ & $\mathrm{PN}$ & Figueroa et al. (2010) & $\mathrm{x}$ & $\mathrm{x}$ \\
\hline Perú & 1994-2011 & $\begin{array}{c}\text { Minería \& } \\
\text { Hidrocarburos }\end{array}$ & $5-9$ & PN & Orihuela (2013) & $\mathrm{x}$ & $\mathrm{x}$ \\
\hline
\end{tabular}

PN: precio neto; $\mathrm{CU}=$ costo del usuario, Elaboración propia

Por un lado, este estudio no incorpora, por ejemplo, la degradación del suelo, la cual podría ser relevante. No todos los estudios incorporan capital humano, sobre todo aquellos publicados durante los 80 s.

El detalle relevante recae en la forma de cálculo del precio sombra. ${ }^{12}$ Los estudios utilizan en su estimación el costo medio como proxy del costo marginal de extracción (es decir, asumen economías de escala en los sectores extractivos). Ello porque resulta muy difícil encontrar o calcular los costos marginales de extracción.

Conforme a los resultados de Orihuela (2012) y EgoAguirre (2012), los dos sectores extractivos considerados (hidrocarburos y minería) presentan economías de escala, en donde el costo medio de extracción es decreciente, de manera que al aumentar el nivel de la producción (extracción) el costo medio se reduce. Por ende, estimar el precio sombra utilizando costo medio de extracción hubiera conllevado a resultados de depreciación natural aun más bajos. ${ }^{13}$

Finalmente, los resultados de las medidas de ingreso de este estudio pueden ser considerados como subestimados ya que no se han incorporado otras formas de capital natural (como ecosistemas).

\section{Conclusiones}

Los resultados demuestran que la medida de ingreso convencional peruana ha estado sobrestimada durante el periodo de análisis. La fracción de sobrestimación osciló entre $4-10 \%$ dependiendo del año y de la medida de ingreso considerada ( $P I B$ o $P N N)$.

\footnotetext{
${ }^{12}$ Cabe mencionar que los precios sombra estimados no tienen una tendencia definida, de manera que no pueden ser considerados como una aproximación de la escasez del recurso natural vinculado

${ }^{13} \mathrm{~A} 1$ no tener evidencia de presencia de economías de escala en los sectores extractivos de otros países, no es factible identificar la dirección del sesgo en sus resultados.
}

Los países pueden mantener su nivel de consumo-per capita solo si ellos pueden acumular stocks de capital renovable a tasas que al menos igualen el agotamiento de los recursos no-renovables (Vincent y Rozali, 2005). Esto parece haber sido el caso peruano, al menos durante el periodo en análisis, aun en términos per-capita. El $P N N$ corregido (verde) creciente evidencia que es posible mantener la tendencia. Sin embargo, esto no excluye la posibilidad que en el futuro cercano no sea posible.

Esto no depende principalmente del agotamiento de los recursos naturales sino del uso de las rentas provenientes de estos recursos. Si estas rentas no son reinvertidas apropiadamente en el largo plazo y si la economía peruana no se ha diversificado, las posibilidades de mantener el consumo se reducirían.

\section{Reinversión de rentas de los recursos naturales}

Las rentas de los recursos naturales no solo deben ser óptimas (es decir, aquellas que maximizan el flujo descontado de ingresos que percibiría el Estado) sino también reinvertidas apropiadamente en otras fuentes de capital. Hay evidencia estas rentas no son optimas, al menos para la minería (Orihuela, 2008). Presumiblemente, aún cuando estas rentas -en parte- son reinvertidas en capital artificial y posiblemente en capital humano, no siendo reinvertidas adecuadamente, lo cual escapa al objetivo de este estudio. ${ }^{14}$ Serían necesarios mayores estudios que corroboren esta hipótesis.

\section{Perspectivas hacia el futuro}

Los recursos naturales más importantes del Perú -en función a su contribución a los ingresos que generan- son los mineros y aquellos que provienen de los hidrocarburos, ${ }^{14}$ Boza (2006) encuentra que las rentas de la minería son destinadas a gastos irrelevantes para el desarrollo de las comunidades en donde el metal es encontrado. 
cuyas rentas son las más significativas. Si las rentas de estos recursos no están siendo -aparentementereinvertidas apropiadamente, ¿Cuál es la implicancia para el crecimiento económico futuro?

Es evidente que los niveles de extracción durante el periodo en estudio, principalmente de metales, han llegado a niveles nunca antes registrados, lo cual sugiere una depreciación acelerada del capital natural no renovable. Es innegable que este agotamiento de los recursos no renovables ha generado al mismo tiempo una mejora en el bienestar y un aporte al crecimiento económico del país, no solo por las reinversiones -escasas- de las rentas de los recursos naturales, si por el efecto multiplicador que -principalmente- el sector minero genera en la sociedad. La cuantificación de este impacto escapa al alcance del estudio aunque seria materia una tarea pendiente.

Este agotamiento debe estar siendo compensado con la incorporación de otras formas de capital a la base productiva o stock total de capital de la economía. Esta hipótesis también debería ser contrastada en futuros estudios.

La riqueza natural peruana depende en gran medida de la riqueza mineral. El agotamiento de reservas probadas es superado una y otra vez con el hallazgo de ricos yacimientos (mayormente en oro, cobre y zinc). Afortunadamente, el Perú es todavía un país muy rico en estos recursos y en la medida que lo siga siendo, el efecto de las (inadecuadas) reinversiones de las rentas de los recursos naturales será opacado. Evidentemente, esto es muy peligroso para el futuro crecimiento de la economía. En conclusión, estamos en la senda óptima del crecimiento (a pesar del agotamiento de los recursos naturales), lo cual es fruto -básicamente- del buen manejo económico, de la favorable coyuntura mundial de precios de los metales y de la riqueza geológica peruana. Así, para potenciar nuestras posibilidades de crecimiento futuro se requiere revisar e implementar una política integral sobre los recursos naturales, y en general de todos los recursos de la economía.

Los efectos de un manejo inapropiado de las regalías de los recursos naturales podría pasarnos la cuenta incluso en el futuro cercano, ya que nuestra economía es muy dependiente de estos recursos. Los futuros gobiernos deben reinvertir estas regalías en otras formas de capital antes que nuestra riqueza geológica empiece a agotarse seriamente o para los momentos en que la minería ya no sea atractiva.Por ahora y afortunadamente, el Perú sigue siendo rico en todo tipo de recursos naturales.

\section{Literatura citada}

Apoyo (2007). Proyecto Camisea: impacto sobre el mercado del gas natural y estimación de los beneficios económicos. Documento elaborado para el Banco Interamericano de Desarrollo. Apoyo Consultoría.
Aronsson, T., Johansson, P. and Lofgren, K.G (1997). Welfare Measurement, Sustainability and Green National Accounting. Chetelham: Edward Elgar.

BCRP (2013). Memoria Anual 2012. Banco Central de Reserva del Perú. Disponible en: www.bcrp.gob.pe. Fecha: febrero 2013

Common, M. and Sanyal, K. (1998). Measuring the depreciation of Australia's non-renewable resources: a cautionary tale. EcologicalEconomics 26: 23-30

Croitoru L. (2010) The cost of environmental degradation. Case Studies from the Middle East and North Africa. The International Bank for Reconstruction and Development/The World Bank. Chapter 3, Air Pollution: The Case of Jordan. Página 37-51.

Ego-Aguirre, M. (2012). Economías de escala del sector petrolero peruano, periodo 1996-2010. Tesis para optar el grado de Magíster Scientiae en Economía de los Recursos Naturales y del Ambiente. Universidad Nacional Agraria La Molina, Escuela de Posgrado.

Figueroa, E.; Calfucura, E. and Nuñez, J. (2002). Green national accounting: the case of Chile's mining sector. Environment and DevelopmentEconomics. 7, pp. 215-239.

Figueroa, E. y Calfucura, E. (2002). Depreciación del capital natural, ingreso y crecimiento sostenible: lecciones de la experiencia chilena. Documento de Trabajo $\mathrm{N}^{\circ} 138$. Banco Central de Chile

Figueroa, E.; Orihuela, C. and Calfucura, E. (2010). Green accounting and sustainability of the Peruvian metal mining sector. ResourcesPolicy (forthcoming).

Hamilton, K. (1994) "Green adjustments to GDP," Resources Policy, vol. 20(3), pp 155-168

Hamilton, K. (1996). "Pollution and Pollution Abatement in the National Accounts". Review of Income and Wealth, Series 42, Number 1.

Hamilton, K. (2000). "Genuine Saving as an Sustainability Indicator". Paper No. 77. Environmental Economic Series, Environmental Department Papers, The World Bank.

Hartwick, J. (1990). "Natural Resources, National Accounting and Economic Depreciation". Journal of PublicEconomics. Vol 43. pp.291-304

Herrera, P. y Millones, O. (2011). ¿Cuál es el costo de la contaminación ambiental minera sobre los recursos hídricos en el Perú? Consorcio de Investigación Económica y Social-CIES-Pontifica Universidad Católica del Perú.

Kröger, T. (2000). Descontaminación atmosférica en Lima Metropolitana: Beneficios en la salud - DIGESA (2000)

Loyola, R. y Soncco, C. (2007). Valoración económica del efecto en la salud por el cambio en la calidad del agua en zonas urbano marginales de Lima y Callao. Consorcio de Investigación Económica y Social -CIES. Disponible en: www.cies.org.pe 
MINEM (2011). Anuario. Disponible en www.minem. gob.pe. Fecha de actualización: Enero 2012.

Miranda, J. (2006). Impacto económico en la salud por contaminación del aire en Lima Metropolitana (Lima Callao). Instituto de estudios peruanos (IEP). Disponible en: http://cies.org.pe/investigaciones/salud/impactoeconomico-contaminacion.

Mota, R.; Domingos, T. and Martins, V. 2010. Analysis of genuine savings and potential green net national income: Portugal 1990-2005. Ecological Economics 69, 1934-1942

Orihuela, C. y Ponce, R. (2004). "Valorando los recursos naturales y su incorporación en las cuentas nacionales". Revista Apuntes, Vol 52, pp. 89-108. Fondo Editorial Universidad del Pacifico.

Orihuela, C. (2008). Sostenibilidad e ingreso del sector hidrocarburos peruano. Consorcio de Investigación Económica y Social-CIES. Disponible en: www.cies.org. pe

Orihuela, 2012. Incluyendo el agotamiento de los recursos naturales en las cuentas nacionales: evidencia peruana del periodo 1994-2011. Informe Final. Informe de Consultoria para la CooperacionTecnica Belga y Consorcio de Investigación Economica y Social (CIES).

Ostro, B. (2004). Outdoor Air Pollution: Assessing the Environmental Burden of Disease at National and Local Levels. Environmental Burden of Disease Series, No. 5.Geneva: World Health Organization.

Pasco-Font, A.; Schroth, E. y McCormick, E. (1996). Ingreso sostenible de la minera peruana. Investigaciones Breves 1. Lima: Consorcio de Investigación Económica y Social. Disponible en: www.cies.org.pe

Repetto, R.; Magrath, W.; Wells, M.; Beer, C. and Rossini, F. (1989). Wasting Assets: Natural Resources in the National Accounts. Washington: World Resources Institute

Repetto, R.; Cruz, W.; Solorzano, R.; de Camino, R.; Woodward, R.; Tosi, J.; Watson, V.; Vasquez, A.; Villalobos, C. and Jimenez, J. (1991). Accounts Overdue: Natural Resources Depreciation in Costa Rica. World Resources Institute. Washington DC.

Sánchez, J. 1998. Estimación de los beneficios en salud del Plan de Descontaminación de Santiago. Estudio encargado por la CONAMA (Comisión Nacional de Medio Ambiente) de la Región Metropolitana

Stauffer, T. R. 1986. Accounting for wasting assests: measurements of income and dependency in oil-rentier states. Journal of Energy and Development 11(1): 69-93

Vincent, J. and Rozali, M-A. 2005. Managing Natural Wealth. Environment and Development in Malaysia. Resources for the Future, Washington DC. Institute of Southeast Asian Studies (ISEAS), Singapore.

Weitzman, M. 1976. On the Welfare Significance of National Product in a Dynamic Economy. Quarterly Journal of Economics. Vol. 90, pp. 156-162
Young, C. E. and Da Motta, R.(----). Measuring sustainable income from mineral extraction in Brazil. ResourcesPolicyVol 21 (2): 113-125. 